\title{
Alcohol Self-administration in Two Rat Lines Selectively Bred for Extremes in Anxiety-related Behavior
}

\author{
Markus S.H. Henniger, Dipl.Biol., Rainer Spanagel ${ }^{\S}$, Ph.D., Alexandra Wigger, Ph.D., \\ Rainer Landgraf, Ph.D., and Sabine M. Hölter, Ph.D.
}

According to the tension reduction hypothesis, individuals with an elevated anxiety level may be more sensitive to the anxiolytic effects of alcohol and may, therefore, have a higher predisposition to consume alcohol. To examine this hypothesis, we studied the drinking behavior as well as the sensitivity to the anxiolytic effect of alcohol in two rat lines that were bred and selected for differences in anxiety-related behavior on the elevated plus-maze: the extremely anxious $H A B$ (high anxiety-related behavior) and the non-anxious $L A B$ (low anxiety-related behavior) lines.

Alcohol self-administration and the occurrence of an alcohol deprivation effect were studied in female and male $H A B$ and $L A B$ rats in a free-choice, 4-bottle home cage paradigm. The sensitivity of $H A B$ and $L A B$ rats to the anxiolytic effect of alcohol was assessed by testing their behavior on the elevated plus-maze after an acute application of ethanol.

During the first days of voluntary ethanol drinking, the ethanol intake and preference of female LABs was significantly higher than that of female HABs. Although not statistically significant, the same trend could be seen in male $L A B s$. Moreover, male as well as female $L A B$ but not $H A B$ rats showed a significant alcohol deprivation effect after abstinence. There were no differences when saccharin was presented to naive animals, indicating that the different ethanol drinking behavior of $H A B$ and $L A B$ rats does not represent a general difference in the consumption of new liquids. Application of ethanol resulted in an anxiolytic effect in HAB but not in LAB rats on the elevated plus-maze.

In summary, increased inborn anxiety and voluntary ethanol consumption of $H A B$ and $L A B$ rats were correlated to some extent; however, this relationship was a negative one. It is concluded that, although such a relationship might exist in some individuals, increased levels of inborn anxiety and alcohol consumption are not necessarily related.

[Neuropsychopharmacology 26:729-736, 2002]

(C) 2002 American College of Neuropsychopharmacology. Published by Elsevier Science Inc.
KEY WORDS: Emotionality; Voluntary ethanol selfadministration; Anxiolysis; Tension reduction hypothesis

From the Max Planck Institute of Psychiatry, Munich, Germany. Address correspondence to: Markus S. H. Henniger, Max Planck Institute of Psychiatry, Kraepelinstr. 2-10, 80804 Munich, Germany, Tel.: + +49 89 30622-641, Fax: + +49 89 30622-569, E-mail: henniger@ mpipsykl.mpg.de

\$Present address: Department of Psychopharmacology, Central Institute of Mental Health, University of Heidelberg, J5, 68159 Mannheim, Germany.

Received July 20, 2001; revised October 18, 2001; accepted October 29, 2001.

Online publication: 11/9/01 at www.acnp.org/citations/ Npp110901204.
The anxiolytic effect of alcohol may be one important motivation for its consumption, at least in individuals who are susceptible to this effect (Pohorecky 1981; Spanagel et al. 1995). It has been argued in the so-called "tension reduction hypothesis" by Conger (1956), that in situations where alcohol consumption is fear-reducing, this effect reinforces alcohol consumption and may, therefore, promote future alcohol intake. This hypothesis predicts that more anxious individuals should benefit more from the anxiolytic effect of alcohol and therefore consume more alcohol than less anxious individuals.

The validity of this concept is still a matter of debate, because its direct assessment is difficult, and only a few 
studies have attempted this. For example, in Wistar rats which were selected from a heterogeneous group for individual differences in anxiety-related behavior on the elevated plus-maze, anxiety levels were positively correlated with alcohol consumption, suggesting a link between emotionality and alcohol consumption (Spanagel et al. 1995). This notion was supported by the finding that bilateral lesions of the central amygdala reduced both experimental anxiety and voluntary ethanol intake (Möller et al. 1997a).

Another approach to the question, whether differences in anxiety-related behavior result in differences in ethanol intake, is the examination of ethanol drinking behavior in animals selectively bred for differences in their inborn emotionality. Until recently, only one such pair of rat strains has been described, the Maudsley reactive (MR) and nonreactive (MNRA) strains (for overview see Broadhurst 1975). These rats were bred and selected for differences in open field defecation which is regarded as a measure of emotional reactivity. However, reports on ethanol drinking in the Maudsley strains are equivocal, some studies demonstrating higher ethanol drinking in the more emotional MR rats (Adams et al. 1991; Brewster 1969; Drewek and Broadhurst 1979; Satinder 1972), and some studies demonstrating the opposite (Brewster 1968; Overstreet et al. 1993; Viglinskaya et al. 1995).

Recently, two Wistar rat lines selectively bred for differences in anxiety-related behavior on the elevated plus-maze have been described, the high anxiety-related behavior (HAB) and low anxiety-related behavior (LAB) rat lines (Liebsch et al. 1998a,b). The difference in anxiety-related behavior between these two lines is evident in both genders and is consistent in further tests of anxiety-related behavior apart from the elevated plus-maze (Henniger et al. 2000; Ohl et al. 2001). Thus, the HAB/ $\mathrm{LAB}$ rat lines represent a valuable tool for the examination of the neurobiology of anxiety and the mechanisms of anxiolytic compounds (Landgraf et al. 1999; Liebsch et al. 1998a,b).

Here we report the examination of voluntary ethanol drinking and of the sensitivity to the anxiolytic effect of alcohol in both HAB and LAB rats.

\section{METHODS}

\section{Animals}

Breeding and selection of the HAB and LAB rat lines was described previously (Liebsch et al. 1998a,b). In brief, adult Wistar rats, obtained from a commercial supplier (Charles River, Sulzfeld, Germany) more than 10 years ago were tested in an elevated plus-maze paradigm (see below) and animals with high (HAB) and low anxiety-related behavior (LAB), respectively, were then mated to establish the two lines. For each new generation, the offspring from both breeding lines was tested on the elevated plus-maze at the age of 10 weeks and the best HAB and LAB breeding pairs were selected. Data presented in this paper were obtained from the generations F5 to F11 (counting was actually started in 1996). All animals were maintained on a 12-hour light/dark cycle (lights on: 6 A.M.) with food and water available ad libitum. If not stated otherwise, rats were kept in groups of up to five individuals.

The experiments were approved by the Committee on Animal Care and Use of the relevant local governmental body.

\section{Experiment 1: Ethanol Self-administration}

Individually housed $\mathrm{HAB}$ and LAB rats (females: 10-13 weeks old, weighing 200-290 g, $\mathrm{n}=7-8$ per line; males: 11-14 weeks old, $300-410 \mathrm{~g}, \mathrm{n}=8$ per line) were given free access to water and ethanol in three different concentrations $(5,10$ and $20 \% \mathrm{v} / \mathrm{v})$ for a period of 11 days. On day 12, the ethanol solutions were removed for 14 days (alcohol deprivation phase, ADP) and then returned for three further days. To avoid development of location preferences, the bottle positions were changed randomly each day. Daily the weights of the bottles containing water and the ethanol solutions, the weights of food as well as the body weights of the rats were determined. From these data, ethanol consumption (in $\mathrm{g} / \mathrm{kg}$ ) and ethanol preference (defined as the percentage share of the sum of consumption from the three ethanol solutions in total fluid consumption) were calculated.

\section{Experiment 2: Saccharin Self-administration}

In order to determine whether the line difference in initial ethanol drinking observed in females was specific for ethanol or may be due to a generally enhanced response of female LAB rats to new liquids, an additional experiment was performed in a new set of animals with a saccharin solution as drinking fluid besides water. After a saccharin dose-response study (with a different set of animals), a saccharin solution was chosen that was clearly preferred over and thus clearly discriminated from water, but did not lead to a ceiling effect, so that possible line differences in saccharin preference would be detectable. The saccharin self-administration experiment was conducted with individually housed, experimentally naive female $\operatorname{HAB}(\mathrm{n}=7)$ and LAB $(\mathrm{n}=11)$ rats $(13-19$ weeks old, weighing 200-300 g). The animals had free access to water and a saccharin solution $(0.01 \% \mathrm{w} / \mathrm{v})$ for 10 days. The position of the two bottles was changed daily.

\section{Experiment 3: Effects of Ethanol on the Elevated Plus-maze Behavior}

Because in Experiment $1 \mathrm{HAB}$ and LAB females differed in their initial ethanol drinking, a new set of fe- 
males of both lines was tested for differences in sensitivity to the anxiolytic effect of ethanol. Female HAB and $\mathrm{LAB}$ rats (14-15 weeks old, weighing 170-270 g) were assigned randomly to two groups per line. Individuals from one group were injected IP with ethanol (1 $\mathrm{g} / \mathrm{kg}$ ), members of the other group were injected with saline. 10 min after the injection, rats were tested in the elevated plus-maze paradigm described previously (Liebsch et al. 1998a,b). Briefly, the plus-maze was made of dark gray PVC and consisted of two open arms $(50 \times 10 \mathrm{~cm})$ and two enclosed arms $(50 \times 10 \times 38 \mathrm{~cm}$ high walls) extending from a central platform $(10 \times 10$ $\mathrm{cm})$. The apparatus was elevated $73 \mathrm{~cm}$ above the floor. The maze, surrounded by an opaque curtain, was lit by two white bulbs above the open arms and two red bulbs above the closed arms (mean light intensity: open arms 140 lux; closed arms 60 lux). At the beginning of a test, each rat was placed on the central platform facing a closed arm. During the 5-min test period behavior was monitored by means of a video camera mounted above the maze and the following measurements were taken by a trained observer: number of entries into open and closed arms (an entry was counted when both forepaws were placed on the respective arm) and time spent on each type of arm. The number of entries into and time spent on the open arms were expressed as percentage of total number of arm entries and total time on all arms, respectively. Before each trial, the maze was cleaned with water containing a detergent and dried with a towel. Animals were brought to the testing room at least $16 \mathrm{~h}$ before testing. The tests were conducted between 8 A.M. and 12.30 P.M.

\section{Drugs}

Solutions for the self-administration experiment were prepared by diluting $96 \%$ pure ethanol with tap water and dissolving saccharin (Sigma, Steinheim, Germany) in tap water, respectively. For IP injections ethanol $(96 \%)$ was diluted with saline to a $12 \%(\mathrm{v} / \mathrm{v})$ solution.

\section{Statistics}

Data obtained from the drinking experiments were analyzed by 3-way and 2-way analysis of variance (ANOVA), respectively. The factors were line $X \operatorname{sex} X$ days and line $X$ days, respectively, with repeated measures for the factor days. The alcohol deprivation effect (ADE) was analyzed by use of 3-way ANOVA (line $X$ ADE $X$ days) with repeated measures for the latter two factors. Elevated plus-maze data were analyzed by 2-way ANOVA (line $X$ treatment). The accepted level of significance was $p<.05$. When appropriate, Newman-Keuls tests were applied for post-hoc comparisons.

\section{RESULTS}

\section{Experiment 1: Ethanol Self-administration}

Figures 1 and 2 show the drinking behavior of male and female HAB/LAB rats. Three-way ANOVA (line $X$ sex $X$ days) revealed significant differences between male and female $\mathrm{HAB} / \mathrm{LAB}$ rats in ethanol intake and in preference during the initial 11-day drinking period (factor sex: $\mathrm{F}_{1,27}=54.6$ (intake), $p<.0001$ and $\mathrm{F}_{1,27}=$ $18.1, p<.001$ (preference), respectively). By use of 2-way ANOVA (line $X$ days), ethanol intake as well as preference in both sexes were examined separately for the first five days and for the second six days of the initial drinking period. While in the first half of the initial drinking period there was a tendency of higher ethanol intake (factor line: $\mathrm{F}_{1,14}=3.5, p=.081$ ) and preference (factor line: $\mathrm{F}_{1,14}=3.4, p=.085$ ) in male LAB compared with male $H A B$ rats, there was no significant difference in the second half (Figure 1). Female LAB compared with $\mathrm{HAB}$ rats displayed an elevated ethanol intake and preference in the first half of the initial drinking period (factor line: $\mathrm{F}_{1,13}=11.7, p<.01$ and $\mathrm{F}_{1,13}=14.7, p<$ .01 ) but not in the second half (Figure 2).

Drinking behavior of male and female HAB/LAB rats three days before and three days after the 14-day ADP was analyzed separately using 3-way ANOVA (line $X$ ADE $X$ days). In males, alcohol deprivation significantly increased ethanol intake as well as preference (factor ADE: $\mathrm{F}_{1,14}=7.3, p<.05$ and $\mathrm{F}_{1,14}=5.4, p<.05$ ). Localization of the differences by post-hoc analysis revealed that only LABs but not HABs had a higher ethanol intake and preference after the ADP compared with all three days before (Figure 1). In females 3-way ANOVA revealed significant differences between $\mathrm{HAB}$ and $L A B$ rats (factor line: $\mathrm{F}_{1,12}=15.8, p<.01$ ) in ethanol intake. Post-hoc analysis revealed that LAB females displayed a higher ethanol intake at the day before and at all three days after the ADP compared with HAB females (Figure 2). Alcohol deprivation significantly increased ethanol intake (factor ADE: $\mathrm{F}_{1,12}=39.3, p<$ .0001) in females. Post-hoc analysis revealed that in HABs there was a significant increase in ethanol intake at the first day after the ADP, while the increase in LABs was highly significant at all three days after the ADP compared with all three days before (Figure 2). Alcohol deprivation significantly increased ethanol preference in females (factor ADE: $\mathrm{F}_{1,12}=10.5, p<.01$ ). Post-hoc statistics revealed that only LABs but not HABs had a higher preference after the ADP than at all three days before (Figure 2).

\section{Experiment 2: Saccharin Self-administration}

As can be seen from Figure 3, there was no significant difference in saccharin preference between female $\mathrm{HAB}$ 

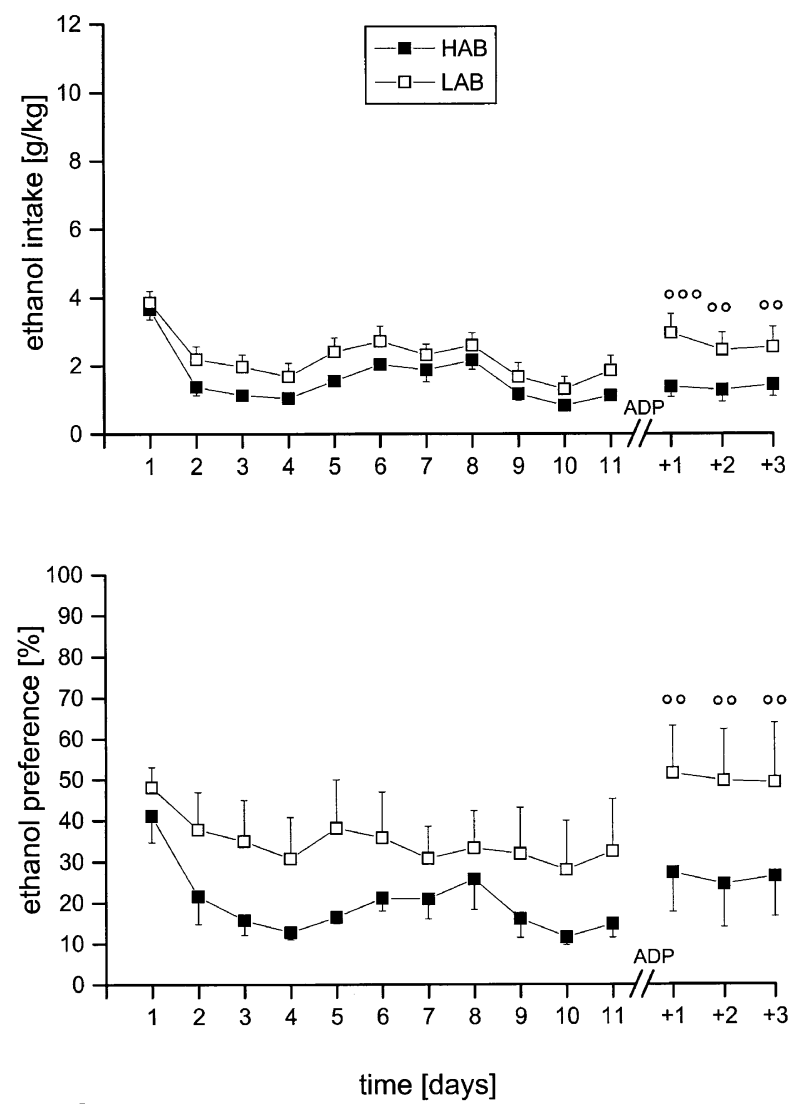

Figure 1. Daily ethanol intake (upper panel) and preference (lower panel) of male HAB and LAB rats during the initial drinking phase and after a 14-day alcohol deprivation phase (ADP). Data points represent the mean absolute ethanol consumption in $\mathrm{g} / \mathrm{kg}$ body weight and the mean ethanol preference as percent intake of all three ethanol solutions per total fluid intake, respectively ( \pm S.E.M.). HAB $=$ high anxiety-related behavior $(\mathrm{n}=8), \mathrm{LAB}=$ low anxiety-related behavior $(\mathrm{n}=8) .{ }^{\circ} p<.01,{ }^{\circ 00} p<.001$ vs. LAB at all three days before the ADP (Newman-Keuls test).

and $\mathrm{LAB}$ rats. Likewise there was no significant difference in saccharin intake or total fluid consumption, respectively (data not shown).

\section{Experiment 3: Effects of Ethanol on Elevated Plus-maze Behavior}

Figure 4 shows the effects of the injection of either saline or ethanol $(1 \mathrm{~g} / \mathrm{kg}$; IP) on behavior of female $\mathrm{HAB}$ and $\mathrm{LAB}$ rats on the elevated plus-maze. HABs displayed a lower percentage of entries into the open arms (factor line: $\mathrm{F}_{1,32}=42.2, p<.0001$ ) as well as percent time spent on them $\left(\mathrm{F}_{1,32}=53.1, p<.0001\right)$ compared with LABs. While ethanol treatment had no significant effect on the percent time spent on the open arms, it resulted in a difference in the percent entries into open
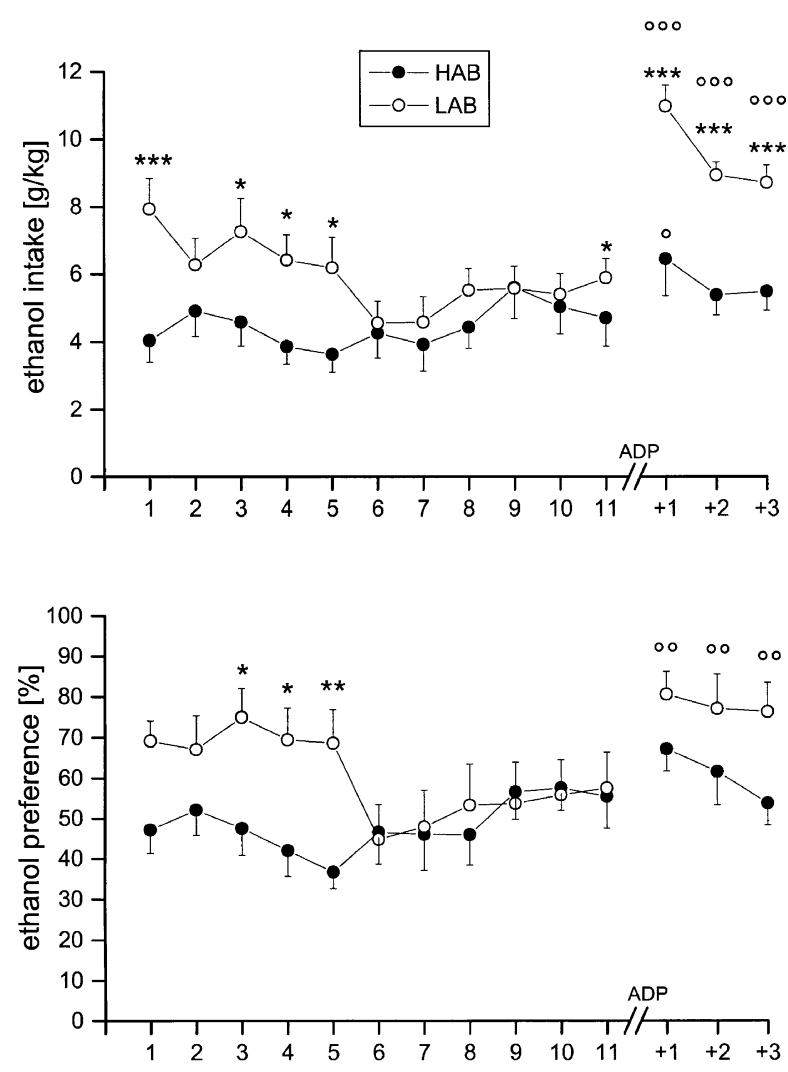

time [days]

Figure 2. Daily ethanol intake (upper panel) and preference (lower panel) of female HAB and LAB rats during the initial drinking phase and after a 14-day alcohol deprivation phase (ADP). Data points represent the mean absolute ethanol consumption in $\mathrm{g} / \mathrm{kg}$ body weight and the mean ethanol preference as percent intake of all three ethanol solutions per total fluid intake, respectively ( \pm S.E.M.). HAB $=$ high anxietyrelated behavior $(\mathrm{n}=8), \mathrm{LAB}=$ low anxiety-related behavior $(\mathrm{n}=7){ }^{*} p<.05,{ }^{* *} p<.01,{ }^{* * *} p<.001 \mathrm{HAB}$ vs. LAB (Newman-Keuls test); ${ }^{\circ} p<.05,{ }^{\circ} p<.01,{ }^{\circ 00} p<.001$ vs. all three days before the ADP (same line; Newman-Keuls test).

arms (factor treatment: $\mathrm{F}_{1,32}=4.9, p<.05$ ). In this parameter, there was also a significant interaction of the factors line and treatment $\left(\mathrm{F}_{1,32}=11.8, p<.01\right)$. Posthoc analysis revealed that ethanol induced a significant increase in open arm entries in HAB but not in LAB rats. Due to a significant difference between the saline treated $\mathrm{HAB}$ rats and the ethanol treated LAB rats $(p<$ .05 , Newman-Keuls test), general ANOVA revealed significant differences in locomotion-measured by the number of closed arm entries-both in the factors line $\left(\mathrm{F}_{1,32}=4.4, p<.05\right)$ and treatment $\left(\mathrm{F}_{1,32}=4.4, p<.05\right)$. However, post-hoc comparison failed to prove significant differences between the same treated animals of the different lines or between the differently treated rats of the same line. 


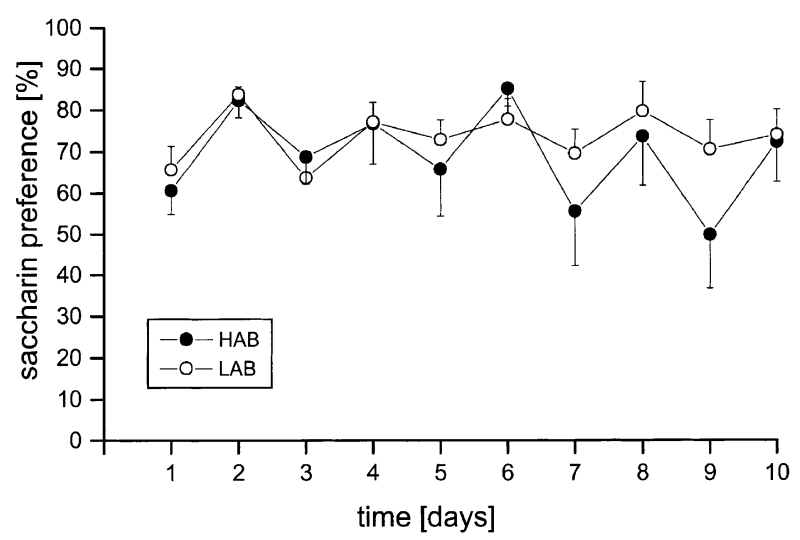

Figure 3. Saccharin preference of female $\mathrm{HAB}$ and $\mathrm{LAB}$ rats during the initial drinking phase. Data points represent the mean saccharin preference as percent of saccharin solution intake per total fluid intake ( \pm S.E.M.). HAB $=$ high anxiety-related behavior $(n=7), \mathrm{LAB}=$ low anxiety-related behavior $(n=11)$.

\section{DISCUSSION}

In this study, we examined the drinking behavior of $\mathrm{HAB}$ and LAB rats in a 4-bottle model of voluntary alcohol drinking in the home cage, which has been extensively used in our laboratory with unselected male Wistar rats (for reviews see Spanagel and Hölter 1999, 2000).

Here, in female individuals, there was a difference in the first five drinking days between the two lines, however-contrasting with the prediction of the tension reduction hypothesis-the less anxious LAB rats consumed more alcohol than the more anxious HAB rats. This initial difference disappeared after five days, but could be seen again after two weeks of alcohol deprivation, when the LAB females, in contrast to HAB rats, showed a clear alcohol deprivation effect.

Although not statistically significant, also male LAB rats tended to consume more ethanol than their $\mathrm{HAB}$ counterparts. The lack of significance might be due to a floor effect, as both male $\mathrm{HAB}$ and $\mathrm{LAB}$ rats consumed significantly less ethanol than females. The phenomenon of a gender-specific difference in ethanol consumption, female rats consuming more than males, has been repeatedly shown in earlier studies (Almeida et al. 1998; Adams et al. 1991; Adams 1995; Li and Lumeng 1984).

Furthermore, the tension reduction hypothesis predicts that, in order to benefit more from alcohol, more anxious individuals should be more sensitive to the anxiolytic effect of alcohol. The elevated plus-maze results indicate that alcohol is anxiolytic in HAB rats as measured by the parameter entries in open arms, but not as measured by the parameter time spent on open arms, which may be considered as more reliable parameter (see File 1992). Thus, alcohol acts as an anxiolytic in
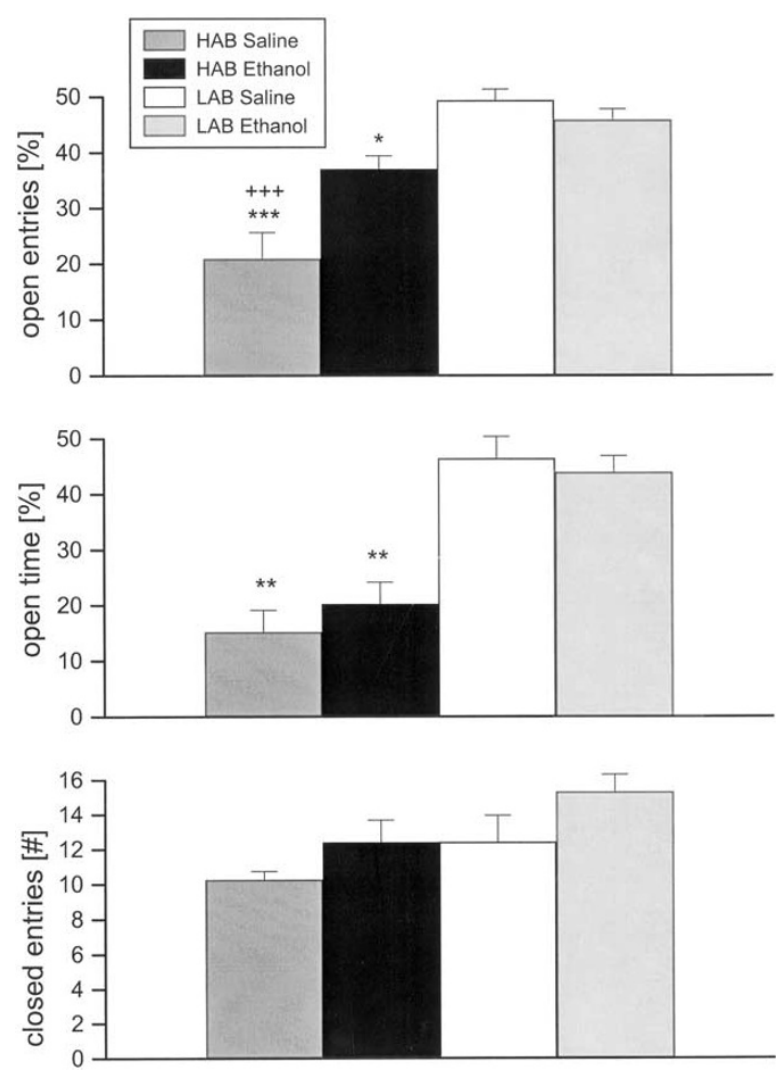

Figure 4. Effects of intraperitoneal ethanol $(1 \mathrm{~g} / \mathrm{kg})$ or saline injections on behavior of female $\mathrm{HAB} / \mathrm{LAB}$ rats in the elevated plus-maze paradigm. Each bar represents open/ total arm entries in percentages (upper panel), open/total arm time in percentages (middle panel) and number of closed arm entries (lower panel), during the 5-min test session (mean + S.E.M.). HAB $=$ high anxiety-related behavior $(n=8$ per group), LAB $=$ low anxiety-related behavior $(n=$ 10 per group). ${ }^{*} p<.05,{ }^{* *} p<.01,{ }^{* * *} p<.001 \mathrm{vs}$. LAB (same treatment), $+++p<.001$ vs. HAB (ethanol treatment), Newman-Keuls test.

HAB rats, but this effect seems to be rather small. In comparison to alcohol, diazepam is a more potent anxiolytic in HAB rats, which acts also as an anxiolytic in LAB animals, though significantly less than in HABs (Liebsch et al. 1998a). The rather small anxiolytic effect of alcohol in these rats might have contributed to the result that the more anxious HAB rats did not consume more alcohol. However, this argument is not supported by a study by Stewart et al. (1993). In this study, two rat lines that were selectively bred for differences in alcohol consumption were tested on the elevated plus-maze. Vehicle-treated alcohol preferring $(\mathrm{P})$ rats displayed higher anxiety-related behavior than vehicle-treated non-preferring (NP) rats. As in this study, the application of the same dose of ethanol $(1 \mathrm{~g} / \mathrm{kg})$ resulted in a very mild decrease of anxiety-related behavior in $\mathrm{P}$, but not in NP rats, and this anxiolytic effect was small rela- 
tive to the anxiolysis produced in both lines by the benzodiazepine CDP (Stewart et al. 1993). In contrast to $\mathrm{HAB}$ rats, $\mathrm{P}$ rats consumed more ethanol than their less anxious non-preferring counterparts.

The present results are in seeming contradiction to a previous study by Spanagel et al. (1995). In that study, those individuals from a heterogeneous group of Wistar rats that displayed the most and those that displayed the least anxiety-related behavior on the elevated plus-maze were selected. The drinking behavior of the 'anxious' rats was compared with that of the 'non-anxious' group. In contrast to the present study, the 'anxious' rats consumed significantly more ethanol than the 'non-anxious' ones. The opposing results might be explained by methodological differences between the two studies: While in our study the rats had the choice between water and ethanol solutions of 5,10 and $20 \%$, in the study by Spanagel et al. (1995) the animals had the choice between water and increasing ethanol solutions ( 2 and $4 \%$, respectively) in a 2-bottle paradigm (see Spanagel and Hölter 1999 for a discussion of the advantages of a 4-bottle model of voluntary ethanol drinking). In this context it is important to note, that only at low ethanol concentrations a positive correlation between anxiety levels and ethanol intake was found, whereas in a follow-up study consumption of higher ethanol concentrations (8 and 10\%, respectively) did not correlate with anxiety-related behavior (Spanagel, unpublished data). It is known that rats usually prefer alcohol solutions of lower $(<6 \%)$ over those of higher concentrations (Meisch and Lemaire 1993). In our study the rats had also the choice of a $5 \%$ ethanol solution, which is comparable to the $4 \%$ concentration in the study by Spanagel et al. (1995). Both, HAB and LAB rats preferred the $5 \%$ over the $10 \%$ and $20 \%$ solution, which is in accordance with previous knowledge about drinking behavior of rats which are not selectively bred for the preference of a 10\% ethanol solution (Spanagel and Hölter 1999; Meisch and Lemaire 1993).

Whereas in the study by Spanagel et al. (1995) selected animals of a "normal" heterogeneous group of rats were used, our study took advantage of two rat lines that were selectively bred for maximum differences in anxiety-related behavior for years. As a consequence, the differences in anxiety-related behavior of $\mathrm{HAB}$ and $\mathrm{LAB}$ rats clearly reflect an inborn trait (see also Wigger et al. 2001), which is less apparent in the rats used by Spanagel et al. (1995). Furthermore, due to selective breeding, the innate anxiety of HAB rats might be increased compared with the 'anxious' rats selected from a heterogeneous group of Wistar rats. Possibly, ethanol consumption and anxiety might be related via an inverted $U$ function, with drinking being more obvious at intermediate levels of anxiety. According to this suggestion, the high innate anxiety of HAB rats might result in a very low approach behavior toward the etha- nol solutions and, thus, to a decreased alcohol intake compared with the 'anxious' rats of the study by Spanagel et al. (1995).

Finally, differences in the sensitivity to the anxiolytic effect of alcohol were not explored by Spanagel et al. (1995). Therefore, it cannot be excluded that the 'anxious' rats in that study were more sensitive to the anxiolytic effect of alcohol than HAB rats in our study.

A central question is why LAB rats consumed more alcohol than HAB rats during the first five days of alcohol exposure and displayed an alcohol deprivation effect, whereas $\mathrm{HAB}$ rats did not. One explanation for the higher ethanol consumption of LAB compared with $\mathrm{HAB}$ rats might be that LABs deal more actively with a new situation than $\mathrm{HAB}$ rats. It has been shown that LABs display a more active coping behavior than HABs when exposed to forced swim stress (Liebsch et al. 1998b). However, the difference in alcohol consumption does-at least in female individuals-not seem to be due to a general difference in the attractiveness of a new liquid between the two lines, as females of both lines did not differ in consumption of a saccharin solution in a two bottle paradigm: water vs. a $0.01 \%(\mathrm{w} / \mathrm{v})$ saccharin solution. Pre-tests had shown that both HAB and LAB rats could clearly distinguish this concentration from water (data not shown).

The most likely explanation for the present results is that anxiety levels and alcohol intake in rats are not closely related factors. Therefore, they might not necessarily be co-selected when animals are selectively bred either for differences in emotionality or for differences in alcohol preference. For example, anxiety levels and alcohol drinking seem to correlate in $\mathrm{SP}$ (sardinian alcohol-preferring) and in Indianapolis P (preferring) rats (Colombo et al. 1995; Stewart et al. 1993), but seem to be less consistently related in the Finnish alcohol-preferring AA (Alko Alcohol) rats (Tuominen et al. 1990; Fahlke et al. 1993; Möller et al. 1997b) and in MR (Maudsley reactive) rats (Brewster 1968, 1969; Adams et al. 1991; Overstreet et al. 1993). This interpretation is supported by a recent factor analysis of 18 behavioral measures from nine pairs of alcohol-preferring and nonpreferring rats (Overstreet et al. 1997). In this factor analysis some behavioral parameters reflecting emotionality (i.e., ultrasonic vocalization and open field defecation), loaded negatively on the factor alcohol. Anxiety- related behavior on the elevated plus-maze, however, was independent of alcohol. These results are supported by a study of Viglinskaya et al. (1995), who stated that the findings in their study "do not provide any evidence for such a consistent relationship between measures in the elevated plus-maze and forced swim test and alcohol intake."

In conclusion, the present results indicate that an inborn increased anxiety-related behavior does not necessarily result in increased intake of or preference for al- 
cohol during "normal," undisturbed home cage drinking conditions. They support the view that anxiety levels and alcohol consumption are not necessarily related. However, one point should be considered. Sitting in the home cage may not be particularly anxiogenic, therefore it may not be a proper situation to experience an anxiolytic effect after consumption of alcohol. Thus, although most studies, including ours, have used home cage drinking, it may not be the best condition to study the relationship between anxiety levels and voluntary alcohol drinking. This possibility may also explain the ambiguous results obtained so far on this issue.

\section{REFERENCES}

Adams N (1995): Sex differences and the effects of tail pinch on ethanol drinking in Maudsley rats. Alcohol 12:463-468

Adams N, Shihabi ZK, Blizard DA (1991): Ethanol preference in the Harrington derivation of the Maudsley reactive and nonreactive srains. Alcohol Clin Exp Res 15:170-174

Almeida OFX, Shoaib M, Deicke J, Fischer D, Darwish MH, Patchev VK (1998): Gender differences in ethanol preference and ingestion in rats-the role of the gonadal steroid environment. J Clin Inv 101:2677-2685

Brewster DJ (1968): Genetic analysis of ethanol preference rats selected for emotional reactivity. J Hered 5:283-286

Brewster DJ (1969): Ethanol preference in strains of rats selectively bred for behavioral characteristics. J Genet Psychol 115:217-227

Broadhurst PL (1975): The Maudsley reactive and nonreactive strains of rats: a survey. Behav Genet 5:299-319

Colombo G, Agabio R, Lobina C, Reali R, Zocchi A, Fadda F, Gessa GL (1995): Sardinian alcohol-preferring rats: a genetic animal model of anxiety. Physiol Behav 57: 1181-1185

Conger JJ (1956): Reinforcement theory and the dynamics of alcoholism. Quart J Stud Alcohol 18:296-305

Drewek KJ, Broadhurst PL (1979): Alcohol selection by strains of rats selectively bred for behavior. J Stud Alcohol 40:723-728

Fahlke C, Eriksson CJ, Hard E (1993): Audiogenic immobility reaction and open-field behavior in AA and ANA rat lines. Alcohol 10:311-315

File SE (1992): Behavioral detection of anxiolytic action. In Elliot JM, Heal DJ, Marsden CA (eds), Experimental Approaches to Anxiety and Depression. Chichester, Wiley, pp25-44

Henniger MSH, Ohl F, Hölter SM, Weißenbacher P, Toschi N, Lörscher P, Wigger A, Spanagel R, Landgraf $R$ (2000): Unconditioned anxiety and social behaviour in two rat lines selectively bred for high and low anxietyrelated behaviour. Behav Brain Res 111:153-163

Landgraf A, Wigger A, Holsboer F, Neumann ID (1999): Hyper-reactive hypothalamo-pituitary-adrenocortical axis in rats bred for high anxiety-related behaviour. J Neuroendocrinol 11:405-407
Li T-K, Lumeng L (1984): Alcohol preference and voluntary alcohol intakes of inbred rat strains and the National Institutes of Health heterogeneous stock of rats. Alcohol Clin Exp Res 8:485-486

Liebsch G, Linthorst ACE, Neumann ID, Reul JMHM, Holsboer F, Landgraf R (1998a): Behavioral, physiological, and neuroendocrine stress responses and differential sensitivity to diazepam in two Wistar rat lines selectively bred for high- and low-anxiety-related behavior. Neuropsychopharmacology 19:381-396

Liebsch G, Montkowski A, Holsboer F, Landgraf R (1998b): Behavioural profiles of two Wistar rat lines selectively bred for high or low anxiety-related behaviour. Behav Brain Res 94:301-310

Meisch RA, Lemaire GA (1993): Drug self-administration. In van Harren F (ed), Methods in Behavioral Pharmacology. Amsterdam, Elsevier, pp 257-300

Möller C, Wiklund L, Sommer W, Thorsell A, Heilig M (1997a): Decreased experimental anxiety and voluntary ethanol consumption in rats following central but not basolateral amygdala lesions. Brain Res 760:94-101

Möller C, Wiklund L, Thorsell A, Hyytiä P, Heilig M (1997b): Decreased measures of experimental anxiety in rats bred for high alcohol preference. Alcohol Clin Exp Res 21:656-660

Ohl F, Toschi N, Wigger A, Henniger MSH, Landgraf $\mathrm{R}$ (2001): Dimensions of emotionality in a rat model of innate anxiety. Behav Neurosci 115:429-436

Overstreet DH, Kampov-Polevoy AB, Rezvani AH, Murelle L Halikas JA, Janowsky DS (1993): Saccharin intake predicts ethanol intake in genetically heterogeneous rats as well as different rat strains. Alcohol Clin Exp Res 17:366-369

Overstreet DH, Halikas JA, Seredenin SB, Kampov-Polevoy AB, Viglinskaya IV, Kashevskaya O, Badishtov BA, Knapp DJ, Mormede P, Kiianmaa K, Li T-K, Rezvani $\mathrm{AH}$ (1997): Behavioral similarities and differences among alcohol-preferring and -nonpreferring rats: confirmation by factor analysis and extension to additional groups. Alcohol Clin Exp Res 21:840-848

Pohorecky LA (1981): The interaction of alcohol and stress. A review. Neurosci Biobehav Rev 5:209-229

Satinder KP (1972): Behaviour-genetic-dependent self-selection of alcohol in rats. J Comp Physiol Psychol 80:422434

Spanagel R, Hölter SM (1999): Long-term alcohol selfadministration with repeated alcohol deprivation phases: An animal model of alcoholism? Alcohol Alcohol 34:231-243

Spanagel R, Hölter SM (2000): Pharmacological validation of a new animal model of alcoholism. J Neural Transm 107:669-680

Spanagel R, Montkowski A, Allingham K, Stöhr T, Shoaib M, Holsboer F, Landgraf R (1995): Anxiety: a potential predictor of vulnerability to the initiation of ethanol self-administration in rats. Psychopharmacology 122: 369-373

Stewart RB, Gatto GJ, Lumeng L, Li T-K, Murphy JM (1993): Comparison of alcohol-preferring $(\mathrm{P})$ and nonpreferring (NP) rats on tests of anxiety and for the anxiolytic effects of ethanol. Alcohol 10:1-10 
Tuominen K, Hilakivi LA, Päivärinta P, Korpi ER (1990): Behavior of alcohol-preferring AA and alcohol-avoiding ANA rat lines in tests of anxiety and aggression. Alcohol 7:349-353

Viglinskaya IV, Overstreet DH, Kashevskaya OP, Badishtov BA, Kampov-Polevoy AB, Seredenin SB, Halikas JA
(1995): To drink or not to drink: Tests of anxiety and immobility in alcohol-preferring and -nonpreferring rat strains. Physiol Behav 57:937-941

Wigger A, Loerscher P, Weissenbacher P, Holsboer F, Landgraf $\mathrm{R}$ (2001): Cross-fostering and cross-breeding of HAB and LAB rats: a genetic model of anxiety. Behav Genet 31:371-382 